\title{
AN INTEGRATED SILICON DOUBLE BRIDGE ANEMOMETER*
}

\section{A F P VAN PUTTEN}

Anmar bv, Sparrenlaan 90,5553CZ Valkenswaard, in cooperation with the Solid State Group, Department of Electrical Engineering, Twente University of Technology, $P O$ Box 217 7500AE Enschede (The Netherlands)

\section{Abstract}

Since 1974, sllicon has been used for making flow sensors, though a lot of problems with respect to drift in time and temperature behavior had to be solved This is especially the case when the chip is operating at elevated temperatures

Heat conduction plays a vital role in the general heat transfer characteristics of the flow sensor Due to a special mounting technique, this conduction has been reduced to less than $6 \%$ of the total power dissipation

To improve drift and temperature behavior, a fundamental attempt has been made to split up the common mode (fault) signal and the differential flow signal This goal is achieved by integrating two equal Wheatstone bridges on one silicon chip and connecting both bridges in opposite ways with respect to each other This results in two flow signals with opposite polarity Compared with a single bridge, reduced offset, a higher signal gain and better stability are achieved The influence of ambient temperature can be compensated completely

A discussion of general heat transfer characteristics is presented, together with results for static and dynamic behavior of the flow sensor

\section{Introduction}

Silicon technology has been applied for making flow sensors since 1974 [1 - 3] A lot of problems relating to drift in time and temperature, offset voltage and accuracy had to be solved after the viable principle of operation was demonstrated A first approach involved a single integrated Wheatstone bridge configuration, which finally resulted in a simple anemometer with acceptable accuracy A lot of time had to be devoted to reducing heat conduction losses Due to a special mounting technique, the final heat conduc-

* Based on a Paper presented at Solıd-State Transducers 83, Delft, The Netherlands, May 31 - June 3, 1983 

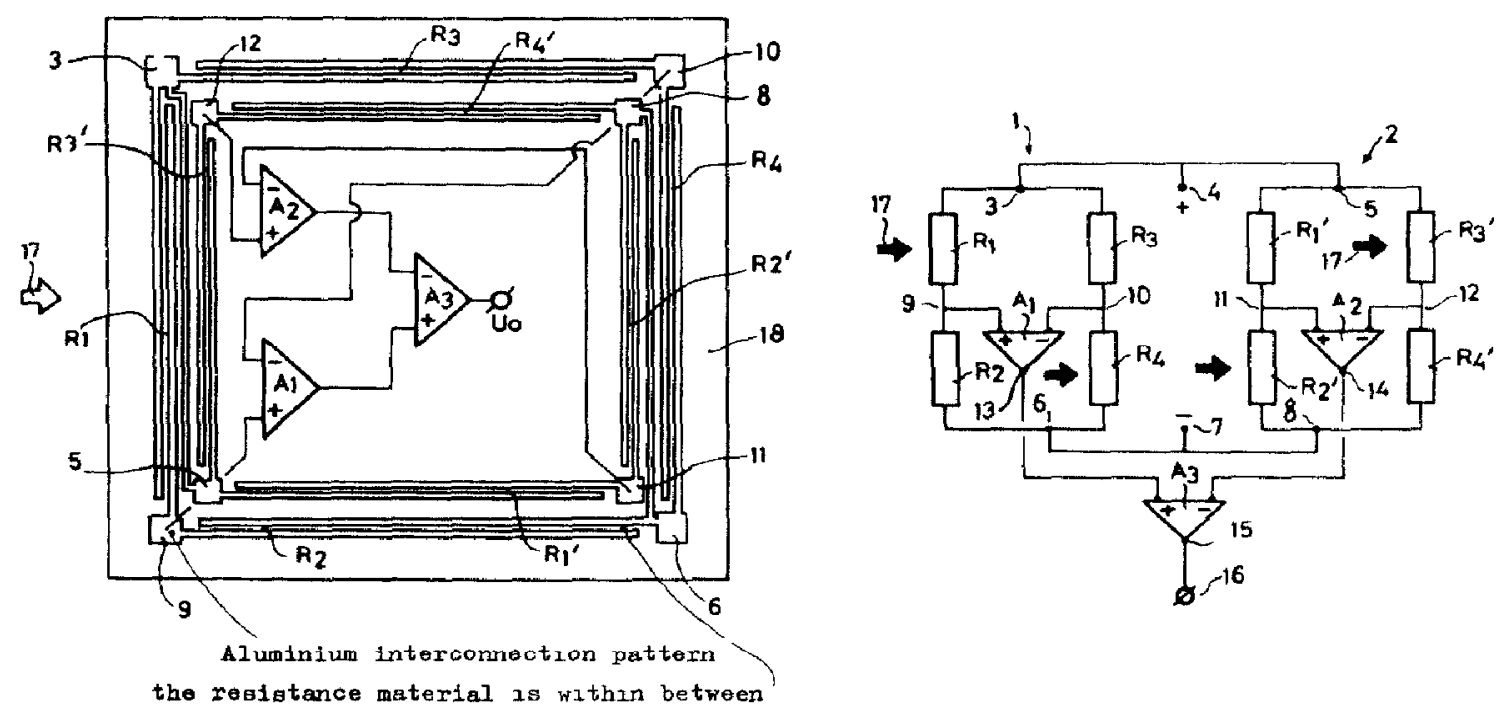

Fig 1 Flow sensor with double bridge configuration (left) and incremental model of the applied circuit (right), demonstratıng that two flow signals with opposite polarity are developed in the same chip

tion is reduced to $6 \%$ of the total power dissipation The result is a very short response time less than $100 \mathrm{~ms}$

A more fundamental attempt has been made to split up the common mode fault signals and the differential mode flow signal This goal could be achieved with a double bridge arrangement Both bridges are connected in an electrically opposite way for flow signals, but the same way for, e g ambient temperature changes (Fig 1) This will be discussed in detall below

Compared with the single bridge, a reduced offset level, an increase in signal level and improved stability with respect to time and temperature are achieved The principle of operation is based on the measurement of temperature differences in a single heated chip of $16 \mathrm{~mm}^{2}$ that are generated when a flund flows along a heated surface The static and dynamic behavior will also be discussed, but general heat transfer characteristics are presented first

\section{Theory of operation}

The principle of operation is based on the measurement of heat transfer differences in a sllicon chip of $4 \times 4 \mathrm{~mm}^{2}$, when a fluid at one temperature flows along the chip surface which is at another higher temperature A thermal-hydrodynamic boundary over the surface will be developed, whose thickness is described by [4]

$\delta_{\mathrm{t}}=\frac{\delta}{\operatorname{Pr}^{1 / 3}}=\frac{50 x}{\operatorname{Pr}^{1 / 3} \operatorname{Re}_{x}^{1 / 2}}$

where $\delta_{\mathrm{t}}=$ thickness of thermal boundary layer, $\delta=$ thickness of hydrodynamic boundary layer, $P r=$ Prandtl number, which is the ratio of the 
kinematic viscosity $\nu$ and the thermal diffusivity coefficient $\alpha$ for the fluid involved For most gases, the Prandtl number is approximately unity (06< $\operatorname{Pr}<10$ ), for air $\operatorname{Pr}=0$ 7) $R e_{x}=$ the local Reynold's number at $x$ (see Fig 2) and is defined as $R e_{x}=\left(V_{\infty} x / \nu\right)$, where $V_{\infty}$ is the free stream velocity It should be mentioned here that due to the mounting technique applied a thermal-hydrodynamic boundary layer beneath the chip's surface is also developed

The applied configuration of the sensors takes full advantage of the difference in thickness of the thermal-hydrodynamic boundary layer (Fig 2) developed over the chip's surface Two Wheatstone bridges designed in a full square are located at the chip's edges with their respective branch resistors

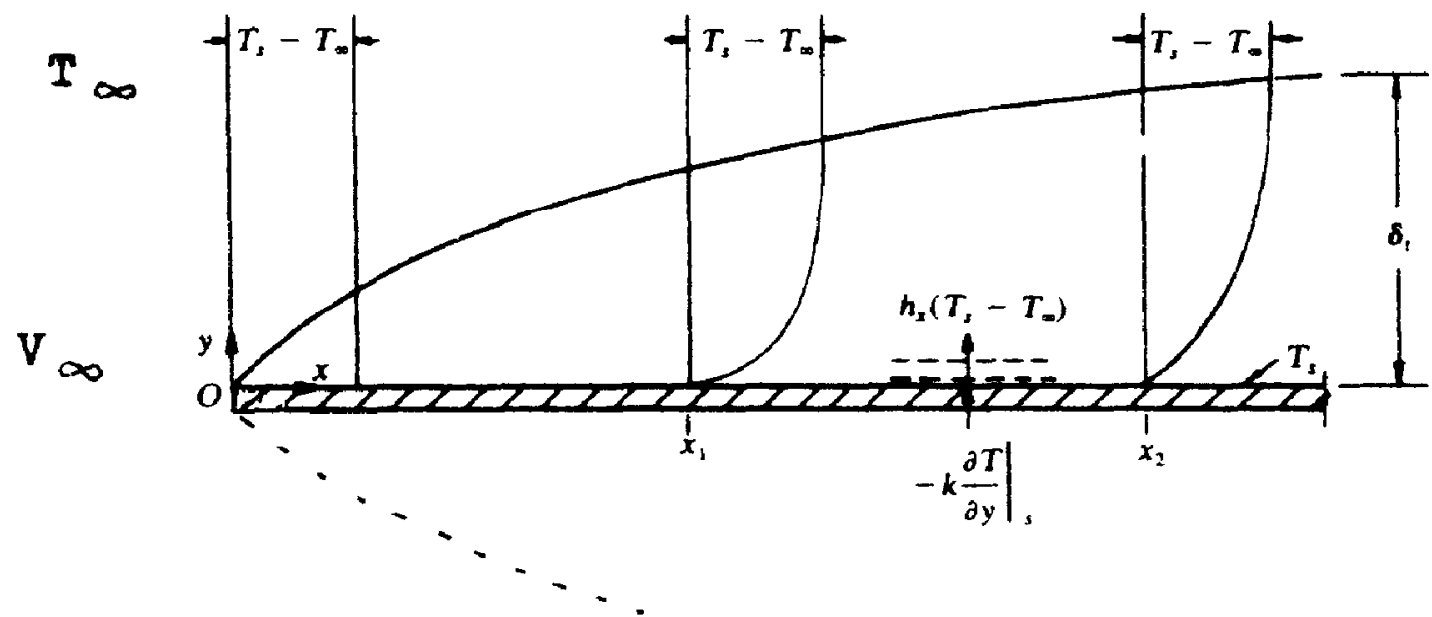

Fig 2 Silcon chip with thermal-hydrodynamic boundary layer

Both bridges are connected in opposition to each other electrically and because of this, they will generate two signals with opposite polarity when a flow is present Because a large length-to-width ratio $(851)$ of all resistors is involved and four resistors are located in parallel with the flow direction and the other four are located perpendicular with respect to the flow direction, the heat transfer is different and both bridges will become unbalanced (see Fig 1) This principle of operation can be understood from the hot wire configuration of which the cooling effect shows a maximum possible directional sensitivity The result will be discussed from an electrical point of view in the next section

The heat balance at the chip's surface is related to the heat conducted from the chip and the heat convected into the flowing fluid This is governed by the equation

$-\left.k \frac{\partial T}{\partial y}\right|_{\mathrm{s}}=h_{x}\left(T_{\mathrm{s}}-T\right)$

where $\partial T /\left.\partial y\right|_{\mathrm{s}}=$ temperature gradient in the $y$-direction, infinite at $x=0$ and zero at $y=\delta_{t}$ as $T=T_{\infty}, k=$ thermal conductivity of the fluid, $h_{x}=$ the local 
heat transfer coefficient, $T_{\mathrm{s}}=$ chip's surface temperature and $T_{\mathrm{o}}=$ free stream fluid temperature

From this equation the local heat transfer coefficient $h_{x}$ and the local dimensionless Nusselt number which describes the local convective heat transfer can be derived From the local Nusselt number $N u_{x}$ an expression for the free stream velocity can finally be found Considering the flat plate behavior and the fixed arrangement of the resistors on the chip, it is more convenient to determine the average heat transfer coefficient and Nusselt number and to account for the drectional sensitivity of this configuration by adding a yaw angle function $\phi(\alpha)$ This is specially useful when a long length-to-width ratio of the resistors is implied Following McCroskey's approach for hot films [5] and including a yaw angle function, the over-all heat transfer for one resistor element can be expressed as

$N u_{\mathrm{f}}=A_{\mathrm{f}}+B_{\mathrm{f}} R e_{\mathrm{f}}{ }^{1 / 2} \operatorname{Pr}^{1 / 3} \phi(\alpha)$

where $A_{\mathrm{f}}$ represents heat conduction to the substrate, as well as other nonflow heat losses $A_{f}$ will be considered as a constant and will finally cancel out of the expression for the free stream velocity, as will be shown Because $R e_{\mathrm{f}}$ is based here on the friction velocity, the exponent of the Reynold's number should be $2 / 3$, for comparison reasons, $1 / 2$ wll be maintained here Finally, the factor $B_{\mathrm{f}}$ is a gauge constant associated with forced convective heat losses and will depend on the applied substrate material, here silicon The yaw angle function proposed by Hinze [6] yields

$\phi(\alpha)=\left(\cos ^{2} \alpha+K^{2} \sin ^{2} \alpha\right)^{\eta}$

For this arrangement $\alpha$ is a constant and is the (yaw) angle between the flow direction and the resistor location For four resistors $\alpha=\pi / 2$ and for the other four resistors $\alpha=0$ The factor $K$ is taken as 02 and $\eta=1 / 4$ Calculation of $\phi(\alpha)$ gives $\phi(\alpha)_{1}=1$ and $\phi(\alpha)_{2}=045$ respectively [5] In a first approximation, the over-all heat transfer differences between the four by four resistor configuration yields

$4\left(N u_{1}-N u_{2}\right)=4(1-045) B_{\mathrm{f}} \operatorname{Re}_{\mathrm{f}}^{1 / 2} \operatorname{Pr}^{1 / 3}$

From eqn (5) it can be seen that $A_{\mathrm{f}}$ cancels out

The non-dimensional forced-convective heat loss difference in eqn (5) represents the power difference per unit length per unit temperature difference The electrical power dissipated per unit length of one resistor is $I^{2} R / l=$ $I^{2} / S \sigma$ and the Nusselt number expressed in electrical quantities for one resistor element is

$N u=\frac{I^{2}-I_{0}^{2}}{S k \Delta T \sigma}$

where $S$ is the cross-sectional area of each resistor, $I_{0}$ is the non-flow value of electric current, $l$ is the lateral dimension of film resistor when $\alpha=0, \sigma$ is the specific electrical conductance and $\Delta T$ is the temperature of the chip above 
adiabatic wall temperature Combining eqns (5) and (6) and putting $\Delta T_{1} \Delta T_{2} \approx T_{\mathrm{s}}{ }^{2}$, the following expression for the flow magnitude is found

$\Delta I^{2}=I_{1}{ }^{2}-I_{2}^{2}=063 B_{\mathrm{f}} P r^{1 / 3} R e^{1 / 2} S k \sigma T_{\mathrm{s}}$

where $\Delta I^{2}$ is the difference of the squared values of the currents due to forced convective heat transfer The indices 1, 2 refer to the four-by-four resistor pairs perpendicular and parallel with respect to the fluid direction and $T_{s}$ is the chip surface temperature

The effects of heat conduction within the substrate dictate maintaining heated-film skin friction gauges at a constant temperature, otherwise $B_{t}$ will change excessively when $\Delta T$ varies Equation (7) can be rearranged into

$\Delta I^{2}=C_{1} V_{\infty}{ }^{1 / 2} T_{\mathrm{s}}$

where $C_{1}=063 B_{\mathrm{f}} \operatorname{Pr}^{1 / 3}(1 / \nu)^{1 / 2} S k \sigma$ Note that $C_{1}$ depends on the ambient temperature, the thermal conductivity $k$, and the kinematic viscosity of the fluid involved in particular varying slightly when the ambient temperature varies It is clear that the forced convective current change is converted into a voltage change due to the configuration used If $T_{\mathrm{s}} C_{1}$ is kept constant, this factor can be considered as the sensitivity of the sensor involved

\section{Desıgn consıderatıons}

The design of the sensor is closely related to the applied principle of operation in which a developed temperature gradient is detected in the sensor itself, when the chip is placed into a moving fluid

The resistor configuration used requires a high temperature coefficient for each resistor The consequence of this is the application of a low doping level, which results in an unwanted high source resistance of the sensor This problem can be solved by connecting the ion-implanted sheet resistance in parallel with the aluminium interconnection pattern, as shown in Fig 1 As is well known, a full symmetry of both bridges offers the highest possible sensitivity of a Wheatstone bridge configuration, and resistors operating at elevated temperature offer better stability than more temperature-sensitive transistor configurations

The applied configuration can be explained as follows from an electrical point of view When the sensor is placed plane-parallel into the moving flund, it can be seen from Fig 1 (left) that the fluid is moving in the direction ind1cated by the arrow (17) When the outer bridge is called 1, it can be seen from the incremental model (Fig 1 ) that resistors $R_{1}$ and $R_{4}$ are cooled more than resistors $R_{2}$ and $R_{3}$ Because a positive temperature coefficient is involved for each resistor, point 9 will rise in voltage and point 10 will fall in voltage level, with respect to the common voltage This signal is amplified by amplifier $A_{1}$ The same story holds for the inner bridge 2, but this shows the reverse generated voltage difference between points 11 and 12 This is due to the opposite electrical connections of both bridges with respect to 
each other Point 3 is connected to point 5 of the inner bridge' In the incremental model, the highest cooling effect for each resistor is indicated by the four arrows in Fig 1 (right) The signal of bridge 2 is amplified by $A_{2}$

It is clear from the incremental model that two flow signals are generated with opposite polarity which differ slightly in amplitude, because the inner bridge has somewhat smaller dimensions than bridge 1

The remaining comon mode fault signals of both bridges, $e g$, ambient temperature changes, will now be cancelled by amplifier $A_{3}$ It is shown that both bridges can be made exactly equal for ambient temperature changes and hence the sensor will be completely independent of ambient temperature influences Amplifier $A_{3}$ delivers the final desired output flow signal

The specific temperature coefficient can be described by $[7,8]$

$\sigma(T)=e p \frac{\mu_{\mathrm{L}} \mu_{1}}{\mu_{\mathrm{L}}+\mu_{1}}$

where $p=$ the applied p-type boron doping concentration, $e=$ specific electron charge, $\mu_{\mathrm{L}}=A(1 / T)^{3 / 2}$ where $A$ is a constant and represents the mobility as a function of lattice vibrations, $\mu_{1}=B T^{3 / 2}$ where $B$ is a constant and represents the mobility of the charge carriers as a function of conized impurities A detalled description of $\sigma(T)$ is beyong the scope of this paper The temperature behavior of four different doped samples is depicted in Fig 3

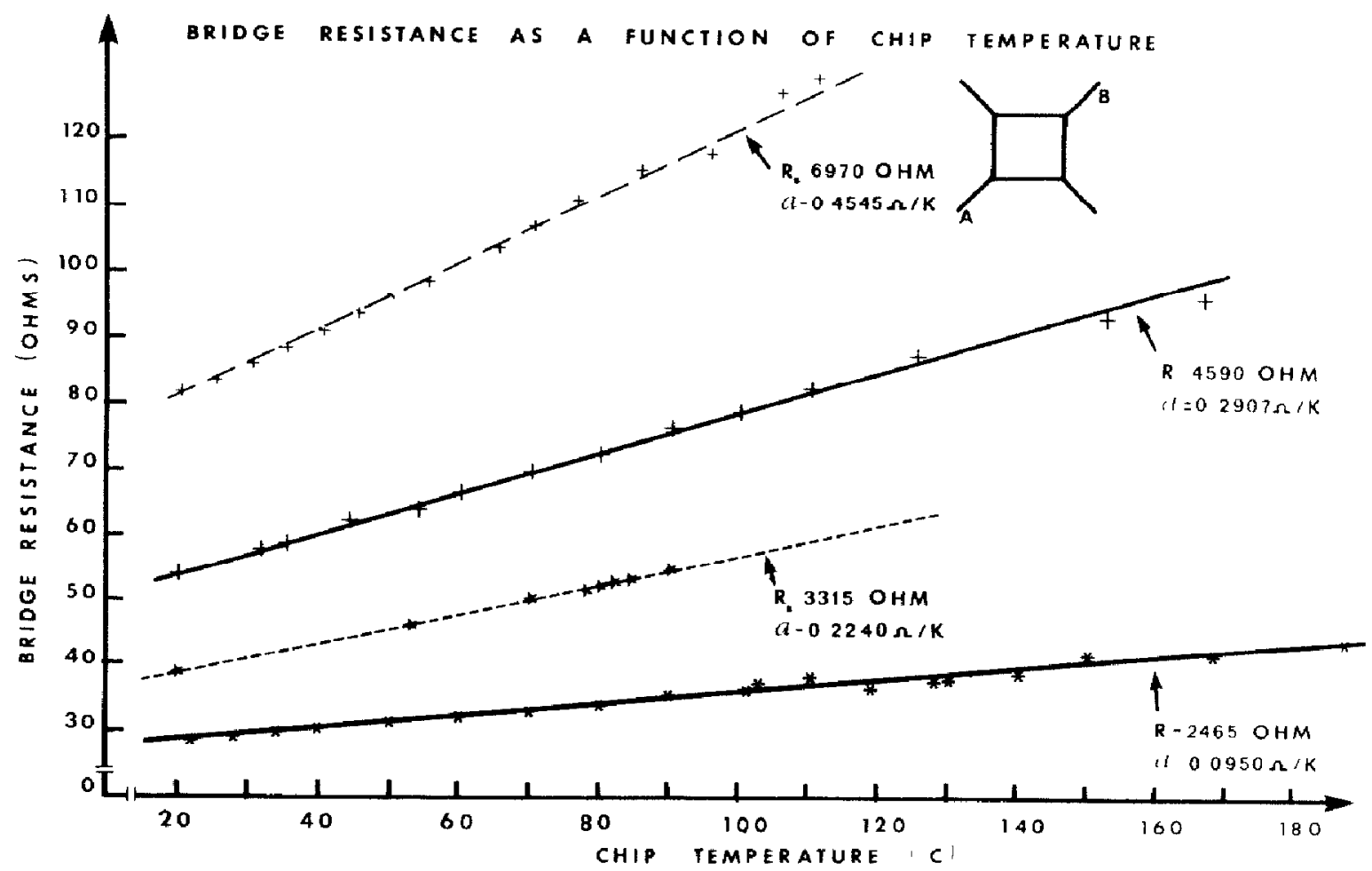

Fig 3 Sensor temperature as a function of power dissipation with doping concentration as parameter 
The chip temperature when no flow condition is presumed can be derived from the expression

$T_{\mathrm{s}}-T_{\mathrm{a}}=D I_{0}^{2} R_{0} /\left(1-\gamma R_{0} D I_{0}^{2}\right)$

where $T_{\mathrm{s}}=$ sensor temperature, $T_{\mathrm{a}}=$ ambient temperature, $D=$ experimental constant determined as $00025(\mathrm{~W} / \mathrm{K})^{-1}$ and $R_{0}=$ resistance value at ambient temperature The measured value of the temperature coefficient $\gamma$ of the applied sample is $0058 \mathrm{~K}^{-1}$ and $I_{0}$ is the supply current under no-flow conditions As already mentioned, despite careful processing small differences in temperature behavior still remain When two bridges are integrated on the same chip it may be expected that the same characteristic faults will occur These faults will show a common mode behavior and can be cancelled by the applied three amplifier configuration The advantages of a double bridge configuration can be summarized as follows

(1) Two bndges with equal low source resistances are avallable, offering a very flexible electronic design The inner bridge is scaled with a fixed scaling of 085 with respect to the outer bridge

(2) Compared with the single bridge configuration, less heating current is required to achieve the desired operating temperature, as can be derived from

$\left.I_{\mathrm{S} 0}^{2} R\right|_{T=T_{1}}=\left.2 I_{\mathrm{D} 0}^{2} R\right|_{T=T_{\mathrm{i}}}$

which results in $I_{\mathrm{D} 0}=I_{\mathrm{So}} / \sqrt{2}$ The static behavior is depicted in Fig 4 , from which it can be seen that a reduced offset voltage is obtained compared with a single bridge powered chip ( $\mathrm{S}=$ single, $\mathrm{D}=$ double for indexing)

(3) A perfect temperature tracking of both bridges under no-flow condition is guaranteed

(4) With a simple offset control, the remaining small differences in temperature behavior can be cancelled completely An example of this behavior is depicted in Fig 5 The step response of both bridges to an ambient temperature change of up to $70^{\circ} \mathrm{C}$ is shown The inner bridge has no correction, the outer bridge shows perfect independence of ambient temperature changes With the same type of offset control, both temperature coefficients can be made equal In general, zero offset voltage does not mean necessarily independence of ambient temperature

(5) Two signals with opposite polanty are avallable due to the arrangement of both bridges, which provides the possibility of splitting up the (flow) differential mode behavior and the (non-flow) common mode behavlor The same figure of merit as is used for operational amplifiers can be used (CMRR)

\section{Measurements and results}

The dynamic measurements are performed in a brass tube of $14 \mathrm{~mm}$ I D For reference purposes, an ordinary industrial standard flowmeter is used 


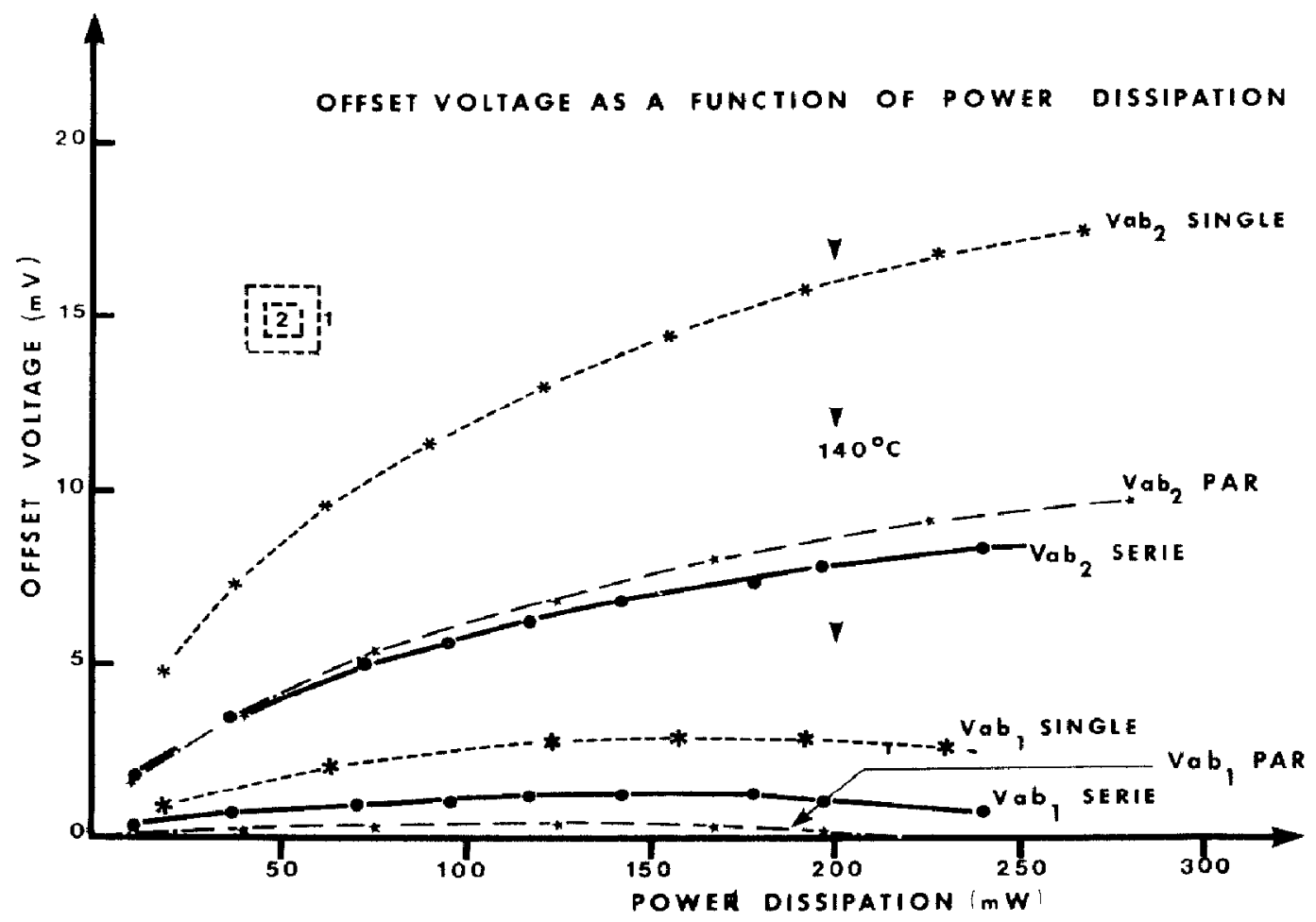

Fig 4 Comparison of offset voltage behaviors for single- and double-bridge heated chip

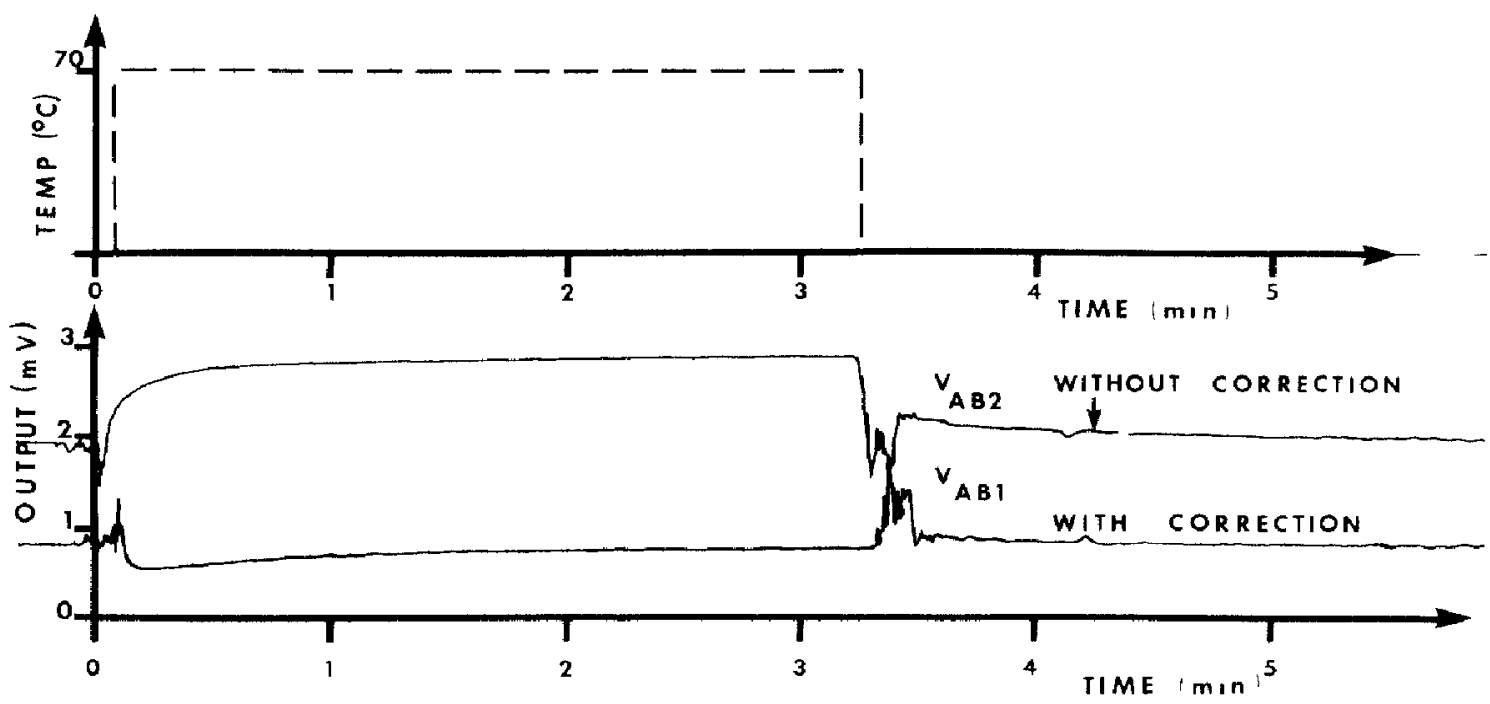

Fig 5 Step response of both bridge output voltages as a function of the ambient temperature under no-flow conditions

The sensor is supplied in voltage source mode and current source mode, only the voltage source mode results being presented here for reasons of space The output of the applied amplifier configuration is simply (see Fig 1) 
$V_{\text {out }}=A_{1} A_{3}\left(V_{\mathrm{ab} 1}+V_{\mathrm{ab} 2}\right)$

1f, for convenience, $A_{1}=A_{2}$ is presumed

The magnitude of the flow is expressed in currents, eqn (8), and can be converted into a voltage quantity which yields the expression

$\Delta V^{2}=C_{2} V_{\infty}^{1 / 2} T_{\mathrm{s}}$

$C_{2}$ is the appropriate constant which can be defined as

$C_{2}=\left.\frac{\partial V_{\text {out }}}{\partial V_{\infty}}\right|_{T_{\mathrm{s}}=\text { constant }}\left(\mathrm{V}^{2}(\mathrm{~m} / \mathrm{s})^{-1 / 2} \mathrm{~K}^{-1}\right)$

The measured values are depicted in Fig 6 It is interesting to note the difference between parallel and series connections of both bridges In parallel connection four currents are involved and symmetry will be more critical with respect to the series connection Moreover, the highest current will flow at the coldest places This mechanism of current dispersion slightly deteriorates the output signal The output signal when the two output voltages of the respective bridges are connected the wrong way to their respective input amplifiers is also depicted If complete symmetry is present, this output signal should be zero and can be used for final correction purposes if

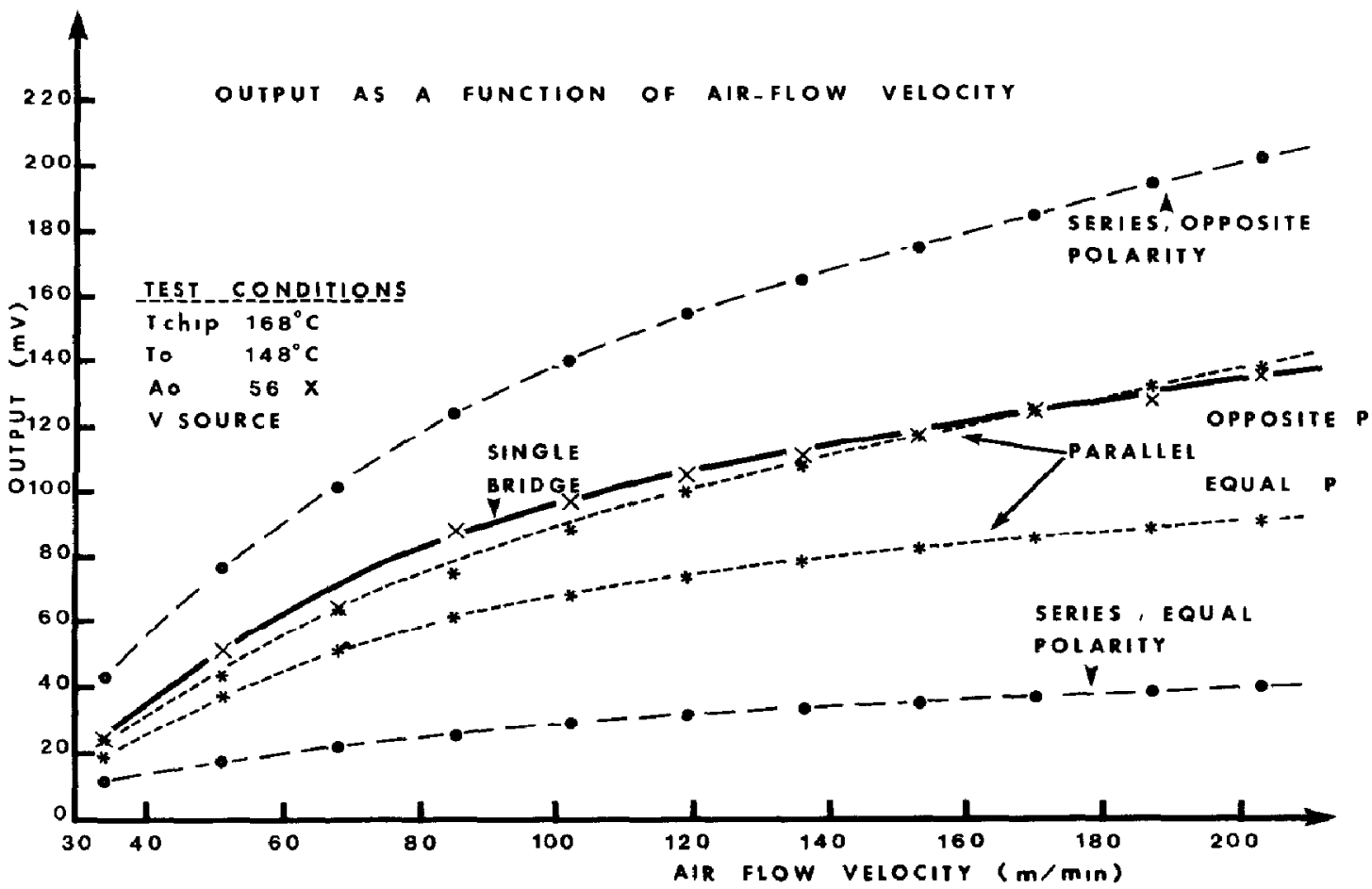

Fig 6 Output voltages of double bridge configuration in series and parallel modes compared with a single bridge sensor Note the difference between parallel and series connections 
required It is interesting to compare eqn (13) with the result given by Rehn et al [9]

\section{Conclusion}

The viability of the double bndge configuration for the measurement of very low flow rates is shown Two signals with opposite polarity are developed when a flow signal is present Common mode signals are highly suppressed and can be compensated fully by applying simple correction curcuitry, giving both bridges the same fault Very probably the same concept could be used for the measurement of other physical phenomena The double bridge configuration facilitates design accuracy by splitting up the differential and common mode signals [10]

\section{Acknowledgements}

The author is greatly indebted to Prof Ir O Memelink and Prof Dr J Middelhoek of the Solid State Group of the Department of Electrical Engineering at the Twente University for providing laboratory facilities The author is particularly grateful to $\mathrm{Mr}$ A Kooy for doing all the processing and for his always enthusiastic assistance

\section{References}

1 A F P van Putten and S Middelhoek, Integrated silicon anemometer, Electrontc Letters, 10 (1974) $425-426$

$2 \mathrm{~J}$ H Huysing, Monolithic flow sensors, a survey, Proc Vaste Stof Sensoren, Conf University of Delft, The Netherlands, Dec, 1980, pp $39-48$

3 A F P van Putten, US Patent 3996 799, Dec 14, 1976

4 D R Pitts and L E Sissom, Heat Transfer, McGraw-Hill, New York, 1977

$5 \mathrm{~W} J$ McCroskey and $E J$ Durbin, Flow angle and shear stress measurements using heated films and wires, Trans ASME, March 1972, $46 \quad 52$

6 J O Hinze, Turbulence, McGraw-Hill, New York, 1959

$7 \mathrm{R}$ Muller, Grundlagen der Halbleıter-Elektronık, Springer-Veriag, Berlın, 1971

$8 \mathrm{~K}$ J Pascoe, Properties of Materials for Electrical Engineers, John Wiley, Chichester, 1973, pp $207-209$

9 L A Rehn, R W Tarpley, K C Wiemer and K M Durham, Dual-element solid-state fluid flow sensor, Soclety of Automotive Engineers, Conf, Detroit, Feb 1980

10 A F P van Putten, Device for measuring the flow velocity of a medium (double bridge anemometer), European Patent Application, March 1983, Nr 82003792 